\title{
Empleo de microdrones para la reconstrucción tridimensional del terreno
}

\section{Using micro-UAV for 3D terrain reconstruction}

\author{
RAMÍREZ-TORRES, José Gabriel †** \& LARRAÑAGA-CEPEDA, Ander \\ Centro de Investigación y Estudios Avanzados del IPN, Unidad Tamaulipas \\ ID 1 ${ }^{\mathrm{er}}$ Autor: José Gabriel, Ramírez-Torres / CVU CONACYT ID: 70840 \\ ID $1^{\mathrm{er}}$ Coautor: Ander, Larrañaga-Cepeda
}

DOI: $10.35429 /$ JCE.2019.9.3.17.27

Recibido 03 de Julio, 2019; Aceptado 30 Septiembre, 2019

\section{Resumen}

Este documento propone un esquema de procesamiento de imágenes que permite la construcción de un modelo digital del terreno empleando elementos tecnológicamente asequibles: una computadora personal y un microdron de bajo presupuesto. Otro punto importante de esta propuesta es que la construcción del modelo no requiere de información georreferenciada $\mathrm{o}$ de sensores sofisticados, pues todo el proceso se basa en el procesamiento de imágenes digitales tomadas con una cámara monocular embarcada en el microdron. El modelo digital del terreno, además de ser construido de manera incremental (al mismo tiempo que se sobrevuela el área), es de una buena precisión, con errores de estimación inferiores a $4 \%$, por lo que el modelo podría emplearse para la planificación de trayectorias y la navegación autónoma del microdron. El propósito de este trabajo es poner al alcance de un gran número de usuarios, tanto particulares como académicos, de una herramienta robusta y asequible para el desarrollo de nuevas aplicaciones basadas en el uso de drones.

Modelo digital del terreno, Microdrones, Procesamiento digital de imágenes

\begin{abstract}
This document proposes an image processing scheme that allows the construction of a digital terrain model using technologically affordable elements: a personal computer and a low-budget micro UAV. Another important issue of this proposal is that the construction of the model does not require georeferenced information or sophisticated sensors, since the whole process is based on the processing of digital images taken with a monocular camera embedded in the microdron. The digital terrain model, in addition to being built incrementally (at the same time the area is overflight), is of good accuracy, with estimation errors of less than $4 \%$, so the model could be used for trajectory planning and autonomous navigation of the micro UAV. The purpose of this work is to provide to a large number of users, both private and academic, a robust and affordable tool for the development of new applications based on the use of UAV.
\end{abstract}

Digital terrain model, Micro UAV, Digital image processing

Citación: RAMÍREZ-TORRES, José Gabriel \& LARRAÑAGA-CEPEDA, Ander. Empleo de microdrones para la reconstrucción tridimensional del terreno. Revista de Ingeniería Civil. 2019. 3-9: 17-27

\footnotetext{
* Correspondencia del Autor (correo electrónico: grtorres@ tamps.cinvestav.mx)

$\uparrow$ Investigador contribuyendo como primer autor.
} 


\section{Introducción}

Un vehículo aéreo no tripulado (UAV, por sus siglas en inglés) es una máquina autopropulsada que emplea las fuerzas aerodinámicas para conseguir la sustentación aérea, que no lleva un operador humano a bordo y que puede volar de manera autónoma o ser controlado de manera remota desde una estación en tierra (Gertler, 2012). Es común llamar "drones" a este tipo de vehículos y si bien cada país clasifica los UAV según características y capacidades definidas por su propia legislación, en este trabajo se considera el empleo de pequeños vehículos menores a 5 $\mathrm{Kg}$, comúnmente llamados "microdrones".

El principal propósito de este documento es presentar las herramientas desarrolladas para realizar representaciones tridimensionales del terreno, empleando elementos fácilmente asequibles: una computadora personal y un microdron equipado con una cámara de video. El aporte más destacable es que, a pesar de lo modesto de los recursos invertidos, el modelo 3D obtenido es de alta calidad y precisión, con información métrica precisa de las elevaciones y estructuras del terreno, incluso cuando no se cuenta con información georreferenciada o señal GPS disponible.

Existen muchas aplicaciones que requieren la representación digital de la superficie de un terreno (DTM, en inglés), tales como la extracción de parámetros terrestres, la descripción del relieve cartográfico y la generación de mapas ortorrectificados (Sirmacek et al, 2010; Weibel y Heller, 1993). Tradicionalmente, estos modelos se obtienen a partir de una secuencia de lecturas con sensores especiales como el telémetro láser (Bybee y Budge, 2015) o cámaras estereoscópias (Haubeck y Prinz, 2013), o a partir de imágenes con información georreferenciada, obtenidas desde avionetas o UAV (Nex y Remondino, 2014; Sanfourche, 2012; Call, 2006; Besnerais et al, 2008).

Asimismo, para que un vehículo (aéreo o no) pueda realizar exitosamente tareas de manera autónoma es necesario que tenga acceso a un mapa tridimensional del medio ambiente, con información precisa sobre distancias y alturas de sus alrededores.
Esta representación debe construirse en tiempo razonablemente corto para que sea útil, pero requiere resolver un conjunto de problemas no triviales como la localización del vehículo sin recurrir a información georreferenciada y la actualización incremental del modelo a partir de la nueva información recabada. En general, estos problemas se abordan empleando sofisticados sensores elemétricos.

El problema bajo estudio puede plantearse de la manera siguiente: Dado un microdron que sobrevuela el terreno, contando con acceso al conjunto de imágenes tomadas por la cámara embarcada y a la información inercial del microdron, generar un modelo 3D del medio ambiente suficientemente preciso que permita estimar la posición, orientación y altitud del microdron, sin recurrir a información GPS.

La propuesta desarrollada consta de tres algoritmos computacionales funcionando en paralelo: un algoritmo para obtener una nube de puntos tridimensionales a partir de una secuencia de imágenes, un algoritmo para estimar el factor de escala real para obtener un modelo métricamente correcto y preciso, y un algoritmo para la construcción de un modelo 3D del medio ambiente. Esta solución requiere que se introduzca un marcador artificial en la escena que indica cuál es el área del terreno de la que se desea construir el modelo digital, además de ayudar al cálculo en línea del factor de escala real del modelo. Un cuarto algoritmo se encarga del control de vuelo del microdron. El modelo tridimensional se construye de manera incremental, a medida que se obtienen más imágenes, con tiempos de respuesta razonablemente cortos en cada actualización. Este enfoque proporciona una base sólida y confiable para la construcción de modelos digitales del terreno, que no requiere de una inversión importante en sensores sofisticados, y que permitiría a particulares y académicos abordar y resolver problemas complejos como la inspección de estructuras, reconstrucción de terrenos y desarrollo de algoritmos de navegación autónoma para vehículos.

\section{Trabajos previos}

En la literatura es posible encontrar múltiples trabajos que abordan la reconstrucción del medio ambiente a partir de imágenes obtenidas con un dron (Nex y Remondino, 2014; Sanfourche, 2012; Call, 2006; Besnerais et al, 2008, 10). 
También, algunos autores sugieren el uso de sensores sofisticados, como múltiples cámaras e información georreferenciada (Krsak et al, 2016; Pollefeys et al, 2008; Barazzetti y Scaioni, 2010; Weiss et al, 2011), cámaras estereoscópicas (Haubeck y Prinz, 2013; Besnerais y Sanfourche, 2008; Wen-Chug y Shu-An, 2008), y dispositivos LADAR (Bybee y Budge, 2015) para obtener la información de profundidad de las imágenes y facilitar la construcción del modelo digital del terreno.

Para la representación tridimensional, algunos autores proponen técnicas que reconocen objetos tridimensionales simples (descriptores de forma) en la escena (Mian et al, 2010; Chen y Bhanu, 2007; Zhong, 2009), pero estas técnicas no son robustas a oclusiones (objetos parcialmente visibles) ni a la presencia de varios objetos similares en la misma escena. Los algoritmos clásicos de Hashing geométrico que se emplean comúnmente en la detección de objetos 3D (Lamdan y Wolfson, 1991) adolescen del mismo tipo de dificultades.

Los enfoques basados en obtener la estructura de la escena a partir del movimiento (Structure-from-motion, SfM) analizan la diferencia entre imágenes para obtener información geométrica de puntos relevantes de las imágenes, obteniendo una nube de puntos tridimensionales geométricamente correcta pero sin información sobre la escala real de la escena, por lo que no es posible calcular las dimensiones absolutas de los objetos (Scaramuzza et al, 2009). Como ejemplos de este enfoque vale la pena mencionar el algoritmo Patch-based Multiview Stereo (PMVS; Furukawa y Ponce, 2010) y el algoritmo A Contratio Structure from Motion (ACSfM; Moulon et al, 2012).

Es posible resolver el problema de estimación del factor de escala real del modelo 3D utilizando información georreferenciada, cuando está disponible (Bybee y Budge, 2015; Nex y Remondino, 2014; Weiss et al, 2011) o introduciendo en la escena una referencia artificial de dimensiones conocidas para calcular el factor de escala durante la construcción del modelo fuera de línea (Davison, 2003). El punto común de todos estos algoritmos es que el tiempo de procesamiento requerido para el emparejamiento de imágenes y el cálculo de la posición y orientación de la cámara respecto a la escena es demasiado alto para aplicaciones de reconstrucción tridimensional en línea.

\section{Propuesta}

Dado que el propósito es desarrollar una herramienta para la construcción de modelos digitales del terreno que requiera elementos tecnológicos asequibles, nos hemos autoimpuesto algunas restricciones materiales: el procesamiento de imágenes se realizará en una computadora personal con conectividad Wi-Fi, el microdron debe ser un dispositivo comercial de bajo costo, sin localizador GPS y con una cámara de baja resolución sin sistema de establización de imagen.

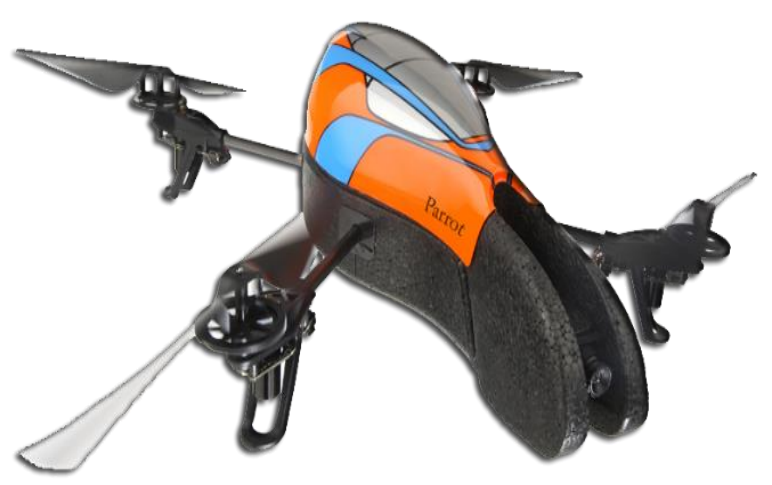

Figura 1 Microdron Parrot AR Drone Fuente: Parrot website

Para el trabajo expuesto en este documento se eligió, por su bajo costo, el microdron Parrot AR Drone (Figura 1) que permite el acceso a los datos inerciales y al flujo de video a través de una conexión Wi-Fi ad-hoc.

Cabe señalar que este microdron es relativamente popular como plataforma experimental para múltiples trabajos de investigación y desarrollo (Bills et al, 2011; Jimenez-Lugo y Zell, 2013; Engel et al, 2012; Soto-Guerrero y Ramírez-Torres, 2013). Para el procesamiento de información, los distintos algoritmos desarrollados se han implementado en una computadora de escritorio iMac con un procesador Intel Core i7.

La solución propuesta está compuesta por cuatro módulos organizados como un flujo de datos (pipeline) para el procesamiento de información: un módulo para el control de vuelo del microdron, un módulo para la generación de nubes parciales de puntos tridimensionales a partir de las imágenes, un módulo para la actualización del modelo 3D del terreno y un módulo para la estimación de escala real del modelo, como se muestra en la Figura 2. 


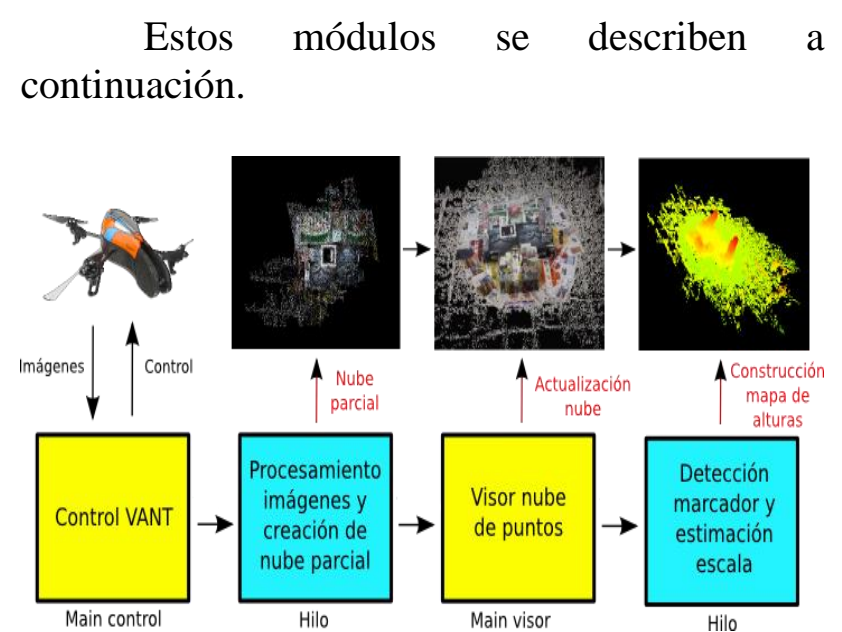

Figura 2 Esquema general propuesto Fuente: Elaboración Propia

\section{Módulo de Control}

Este módulo permite controlar el microdron de manera manual o empleando una trayectoria preprogramada. Consiste básicamente en el autómata descrito en el Algoritmo 1, que mantiene la comunicación entre la computadora personal y el microdron, analiza el flujo de imágenes de la cámara en la búsqueda del marcador artificial para alimentar el pipeline de construcción del modelo $3 \mathrm{D}$, y recibe la información inercial del vehículo para estimar la trayectoria actual del dron, empleando el cálculo de odometría descrito en el Algoritmo 1A.

El Algoritmo 1 utiliza un marcador artificial del tipo ArUco, que es una imagen cuadrada con información binaria, detectado fácilmente y de manera rápida y robusta, y es empleado para calcular la matriz de pose de la cámara (la matriz de transformación homogénea que contiene la información de la ubicación y orientación de la cámara con respecto al centro del marcador artificial).

Al introducir un marcador artificial en la escena se obtienen tres beneficios: 1) permite calcular la posición del microdron respecto a la escena en tiempo real, con poca sobrecarga computacional; 2) dado que se conocen las dimensiones del marcador, es posible estimar el factor de escala real de la escena; y 3) permite establecer el plano XY del modelo construido.

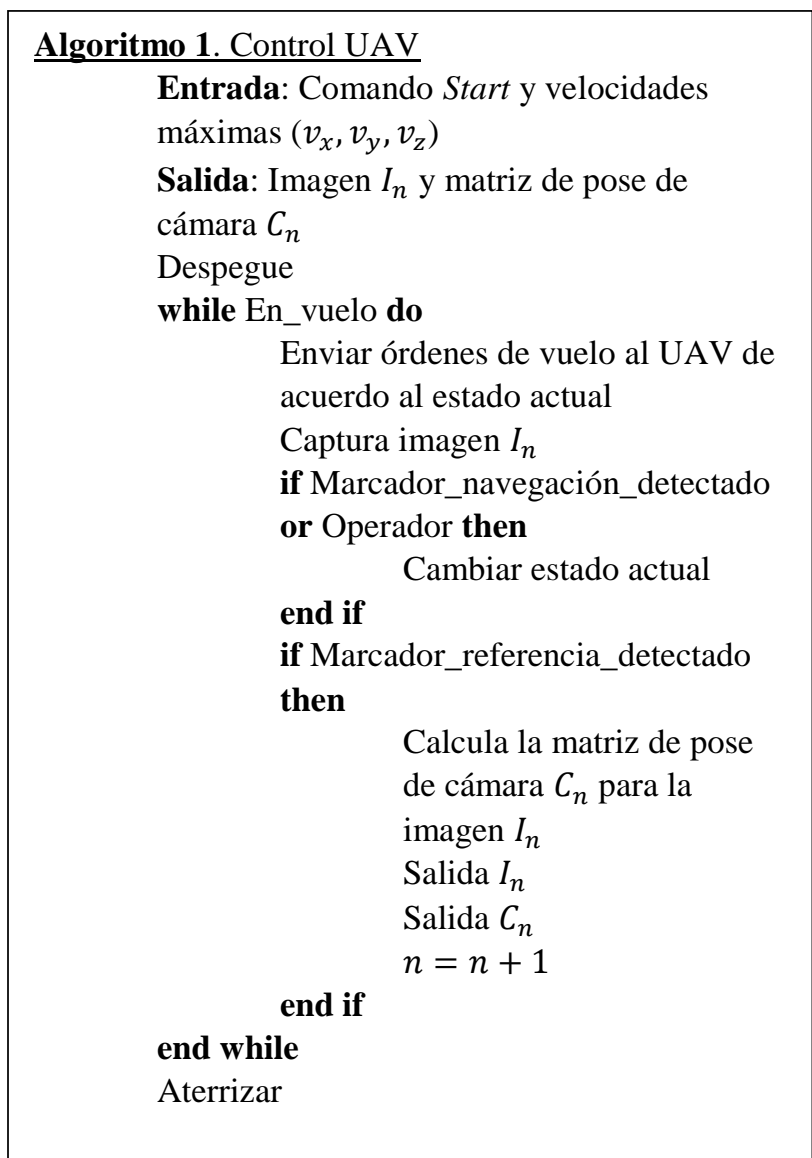

Algoritmo 1 Módulo de Control

\begin{tabular}{|c|}
\hline Algoritmo 1a. Odometría del UAV \\
\hline Entrada: Datos inerciales: velocidades \\
$\left(v_{x}, v_{y}, v_{z}\right)$ y ángulos yaw, pitch, roll \\
Salida: Posición estimada $P=(x, y, z)$ \\
$t_{k-1}=t_{k}=$ tiempo_actual \\
$P=(0,0,0) ;$ Posición inicial \\
while En_vuelo do \\
$t_{k}=$ tiempo_actual \\
Recibir datos inerciales \\
$($ velocidades, ángulos $)$ \\
$d t=t_{k}-t_{k-1}$ \\
$M=\left[v_{x} d t, v_{x} d t, v_{z} d t\right]$ \\
Cálculo de matrices de rotación \\
$R_{y a w}, R_{p i t c h}, R_{\text {roll }}$ \\
$P=P+R_{\text {yaw }} R_{\text {pitch }} R_{\text {roll }} M$ \\
$\quad$ Nueva posición estimada \\
$t_{k-1}=t_{k}$ \\
end while
\end{tabular}

Algoritmo 1a. Módulo de Odometría

\section{Módulo de Procesamiento de Imágenes y Generación de nube parcial}

Este módulo es el primero del pipeline de procesamiento de información para la construcción del modelo 3D. 
Recibe todas las imágenes en las que aparece el marcador artificial, las organiza en paquetes de 3 imágenes sucesivas y utiliza una adaptación del algoritmo PMVS (Furukawa y Ponce, 2009) para construir una nube parcial de puntos tridimensionales por cada paquete, como se describe en el Algoritmo 2.

Cada nube parcial de puntos contiene las coordenadas tridimensionales de los puntos de interés detectados en cada paquete de 3 imágenes únicamente, independientemente de los demás paquetes.

Algoritmo 2. Cálculo de la nube parcial de puntos

Entrada: Imágenes $I_{1}, I_{2}, I_{3}$ y sus

correspondientes matrices de pose de cámara $C_{1}, C_{2}, C_{3}$

Salida: Nube parcial de puntos parcial, con localización 3D $(x, y, z)$ y color $(R, G, B)$ parcial $=\varnothing ;$ Nube de puntos inicial for cada imagen $I_{i}$ do

Detección del conjunto de $\mathrm{POI}_{i}$, usando detector de Harris Cálculo del conjunto de end for descriptores $F_{i}$

parcial $=\operatorname{PMVS}\left(F_{1}, F_{2}, F_{3}, C_{1}, C_{2}, C_{3}\right)$

Algoritmo 2 Módulo de Cálculo de nubes parciales

En esta adaptación, el algoritmo PMVS ha sufrido algunas modificaciones para acelerar su tiempo de respuesta, en detrimento del número de puntos tridimensionales construidos: las imágenes se analizan en bloques de 3 imágenes consecutivas, la búsqueda de puntos de interés (POI) en cada imagen utiliza un detector de esquinas de Harris (Harris y Stephens, 1988) y emplea un algoritmo de "vecinos más cercanos", para localizar rápidamente los puntos consistentes con la transformación epipolar entre imágenes. Los puntos consistentes en las tres imágenes se emplean para determinar su localización tridimensional en la escena a través de un proceso de triangulación, formando una nube parcial de puntos tridimensionales.

En promedio, cada bloque de tres imágenes es procesado en menos de un segundo.

\section{Módulo de Actualización incremental del modelo 3D}

En este módulo, cada una de las nubes parciales de puntos construidas en el módulo anterior es comparada con el modelo 3D actual, para encontrar el mejor emparejamiento 3D y actualizar el modelo digital de la escena. En efecto, cada una de las nubes parciales de puntos es independiente de las otras, con su propia escala y orientación, por lo que no es posible realizar una fusión directa entre ellas.

Para corregir las coordenadas de cada nube parcial, de manera que sea coherente con el modelo 3D de la escena actual, se emplea un procedimiento de votación por Transformada de Hough para objetos 3D (Hough, 1962), aprovechando la superposición entre la nube parcial de puntos y el modelo 3D del terreno, como se describe en el Algoritmo 3.

El procedimiento es como sigue: cada una de las nubes parciales es muestreada de manera uniforme en pequeños cuboides, definidos por sus centroides, calculando el vector normal a la superficie en cada uno. Cada región es descrita localmente empleando un descriptor tipo SHOT (propuesto por Tombari et al, 2010, que emplea un histograma de orientación para describir localmente una región 3D).

Estos descriptores se comparan uno a uno para determinar los emparejamientos con un distancia euclidiana (suma de diferencia de cuadrados) inferior a un valor umbral (llamado umbral Hough) predeterminado.

Este valor de umbral tiene un impacto en la precisión geométrica y en la densidad (cantidad) de puntos del modelo 3D del terreno. 


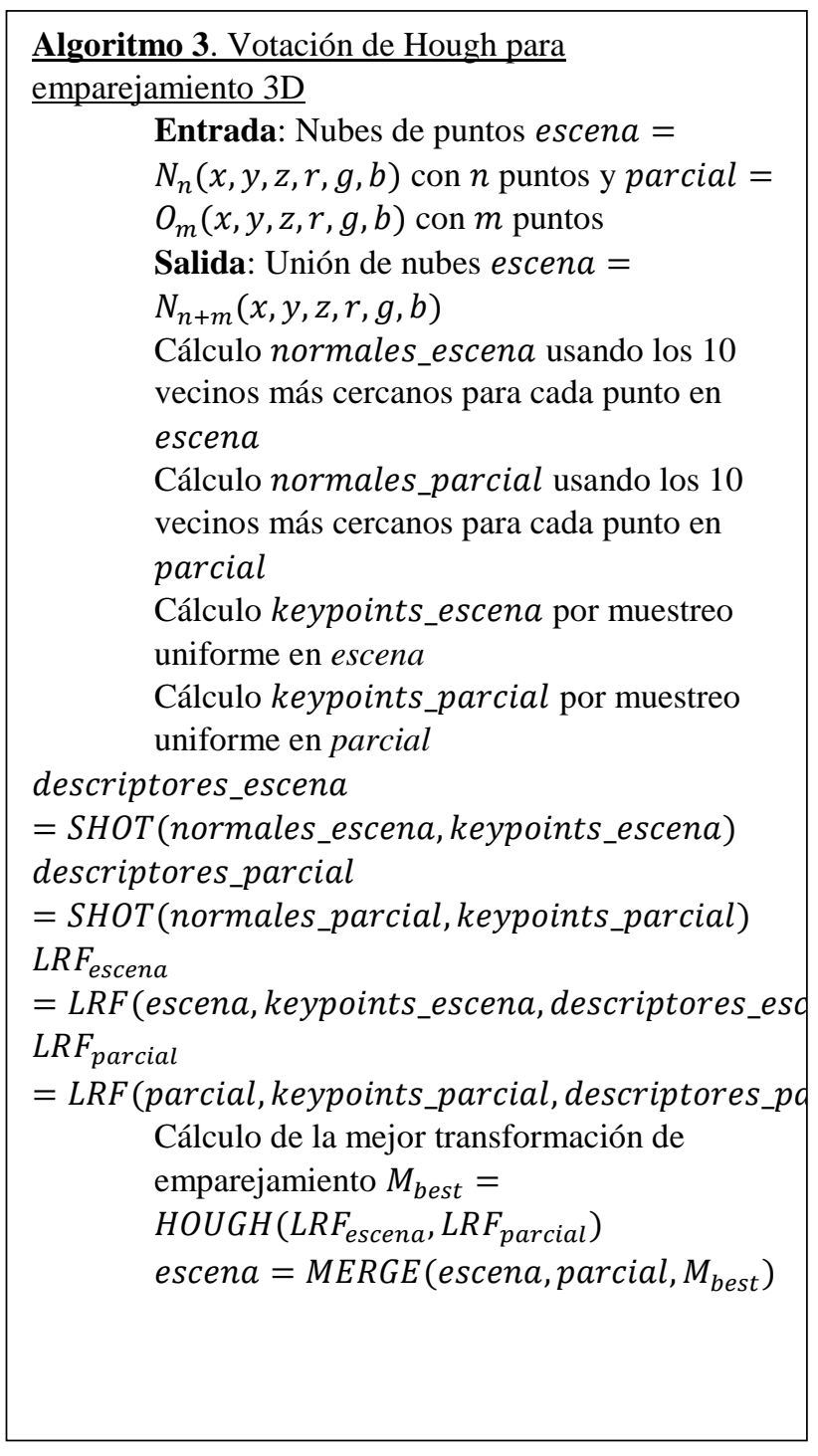

Algoritmo 3 Módulo de Emparejamiento 3D

Finalmente, una adaptación del algoritmo BOARD (Petrelli y Stefano, 2011) asigna un marco de referencia local (LRF) tanto para la nube parcial como para el modelo 3D actual, que permite establecer, con el método de votación de Hough, la mejor matriz de transformación para lograr el emparejamiento entre los dos.

Este proceso se repite con cada nube parcial construida a partir de un bloque de tres imágenes sucesivas, lo que permite realizar una construcción incremental del modelo digital del terreno, al mismo tiempo que el microdron sobrevuela el medio ambiente.

\section{Módulo de Estimación de Factor de escala}

Finalmente, este módulo analiza el modelo digital 3D del terreno para localizar de manera precisa el marcador artificial y emplea las medidas del mismo (conocidas a priori) para corregir el factor de escala del modelo completo, empleando el mismo sistema de votación de Hough del módulo anterior. De esta manera, se resuelve el problema de certidumbre de escala para obtener un modelo 3D del medio ambiente métricamente correcto.

\section{Resultados en condiciones controladas}

Para probar la funcionalidad del esquema propuesto, se construyeron en laboratorio diferentes maquetas que fueron sobrevoladas con el microdron para obtener el modelo digital del terreno. Algunos ejemplos pueden verse en la Figura 3. Para los propósitos de este documento, presentaremos los resultados obtenidos con la maqueta mostrada en la Figura 4. Se realizaron 35 experimentos (sobrevuelos) sobre esta maqueta, a una altura aproximada de $2 \mathrm{~m}$, mientras se capturaban imágenes con la cámara embarcada (entre 60 y 105 imágenes, con una resolución de $640 \times 480$ pixeles) para cada sobrevuelo.

Estos datos permitieron validar la precisión métrica del modelo obtenido, realizando un análisis estadístico de cuatro medidas de referencia (A, B, C y D en la Figura 4) conocidas de la maqueta.

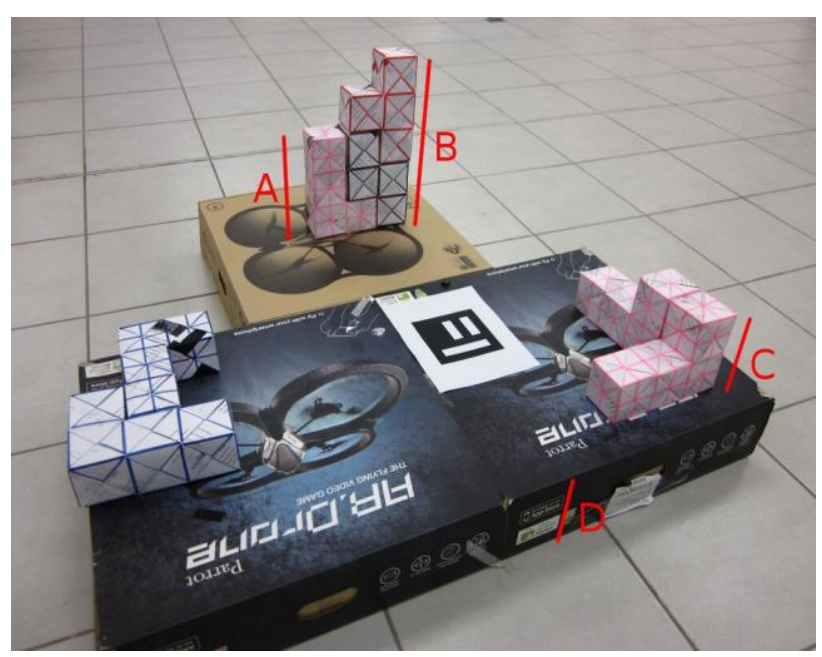




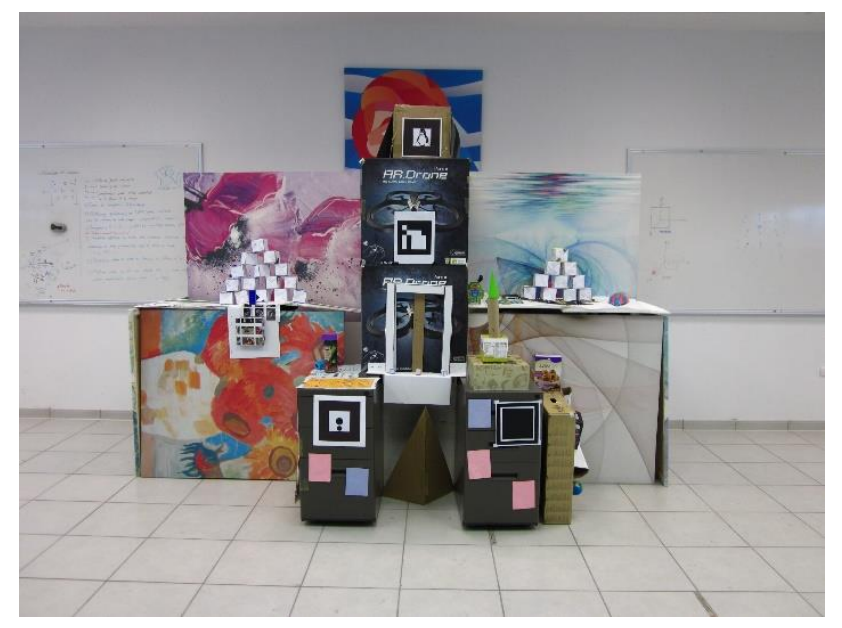

Figura 3 Maquetas elaboradas para pruebas en condiciones controladas

Fuente: Elaboración Propia

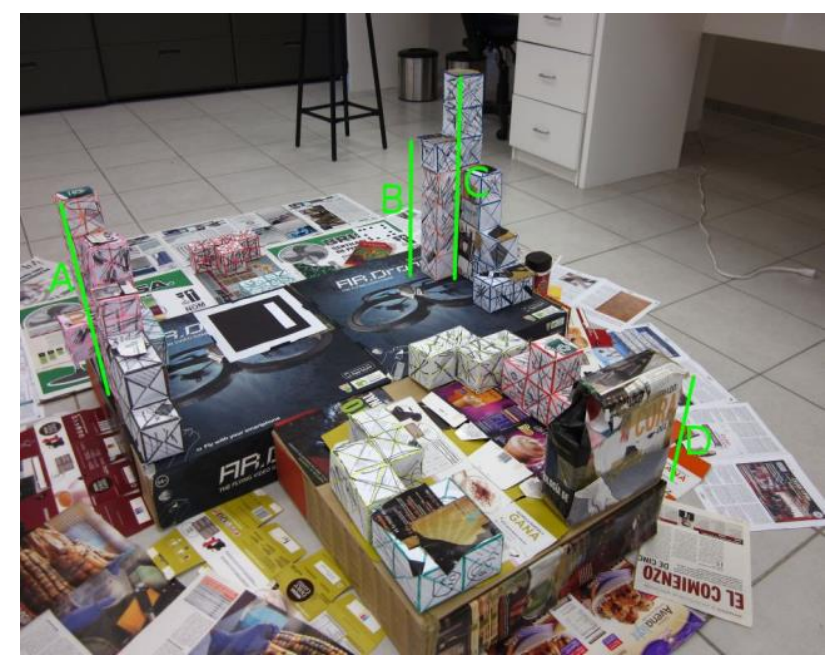

Figura 4 Maqueta para experimentación Fuente: Elaboración Propia

La Figura 5 muestra uno de los modelos digitales obtenidos durante la experimentación, donde el color amarillo indica los puntos que se encuentran a la misma altura que el marcador, el color verde indica los puntos por debajo del marcador y el color rojo los puntos por encima del marcador. Se puede observar que la maqueta está contaminada por algunos puntos atípicos obtenidos durante la reconstrucción.
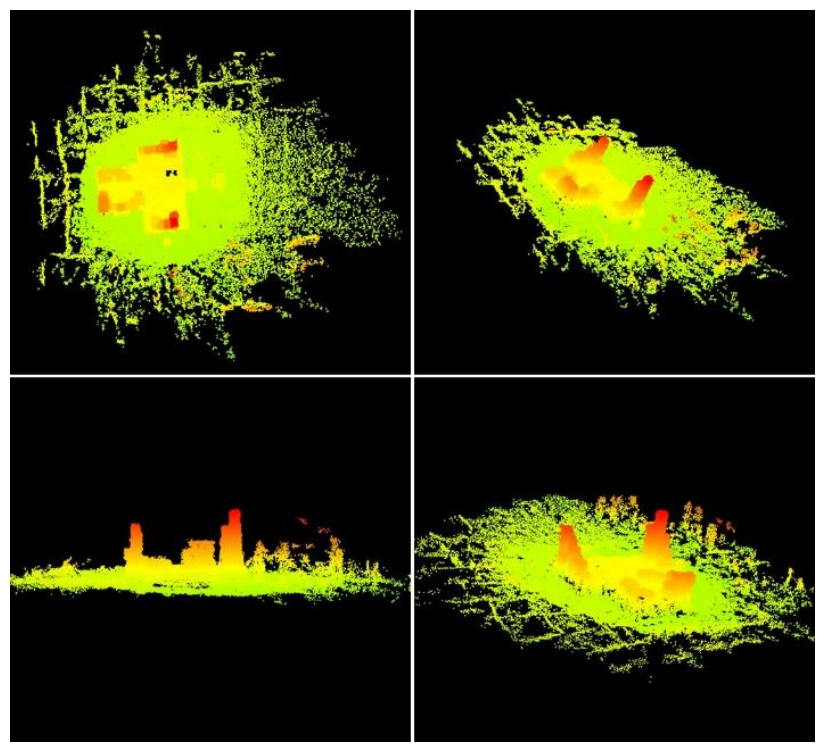

Figura 5 Modelo digital obtenido Fuente: Elaboración Propia

Los resultados del análisis estadístico se resume en la Tabla 1, con los valores promedios estimados para cada una de las alturas de referencia consideradas, para diferentes valores de umbral Hough empleado en el proceso de emparejamiento. Es importante recordar que el módulo de Actualización incremental del modelo 3D requiere de un parámetro de umbral Hough para lograr el emparejamiento de cada nube parcial de puntos con el modelo.

Este parámetro tiene un impacto importante en la calidad del modelo obtenido: si el valor es muy pequeño, el emparejamiento es muy estricto y por ende el número de emparejamientos válidos se reduce, con lo que la densidad de los puntos del modelo se reduce significativamente; si el valor es muy alto, el emparejamiento es más tolerante y el número de emparejamientos considerados válidos aumenta, lo que introduce puntos con un mayor error de posición o totalmente espurios. En consecuencia, es necesario encontrar un compromiso entre densidad y precisión del modelo 3D deseado.

\begin{tabular}{|c|c|c|c|c|c|}
\hline $\begin{array}{l}\text { Umbral } \\
\text { Hough }\end{array}$ & $\begin{array}{c}\text { Altura } \\
\text { A } \\
(0.390 \\
\text { m })\end{array}$ & $\begin{array}{c}\text { Altura } \\
\text { B } \\
(0.395 \\
\text { m) }\end{array}$ & $\begin{array}{l}\text { Altura } \\
\text { C } \\
(0.550 \\
\mathrm{m})\end{array}$ & $\begin{array}{c}\text { Altura } \\
\text { D } \\
(0.240 \\
\mathrm{m})\end{array}$ & $\begin{array}{c}\text { Error } \\
\text { relativo } \\
\text { promedio } \\
(\%)\end{array}$ \\
\hline 0.010 & 0.4195 & 0.4098 & 0.5645 & 0.2471 & 4.22 \\
\hline & 0. & 0.4039 & $5 / 01$ & 1 & 4.41 \\
\hline & & 0.4 & $T$ & 5 & 6.70 \\
\hline & & & 0.5111 & 0.2513 & 7.09 \\
\hline 0.100 & 0.5171 & 0.4985 & 0.7001 & 0.3071 & 28.51 \\
\hline
\end{tabular}

Tabla 1 Resultados de estimación del modelo digital obtenido

Fuente: Elaboración Propia 
Por lo que respecta a los tiempos de cómputo, emplando una computadora de escritorio con un procesador Intel Core i5 y 4 GB de memoria RAM, el esquema propuesto analiza y actualiza el modelo tridimensional cada 1.05 segundos en promedio, lo que significa que procesa 3 imágenes por segundo.

Esta velocidad de procesamiento es compatible con una aplicación de tiempo real del esquema propuesto, ya que las imágenes deben ser lo suficientemente distintas entre sí para poder triangular la posición de los puntos del modelo.

\section{Resultados en escenarios realistas}

Para evaluar el desempeño del esquema propuesto en condiciones más realistas y naturales, se colocó el marcador artificial en un ambiente de corredor, con una escalera con un pasamano tubular, iluminado con luz solar a través de ventanales, mostrada en la Figura 6. La Figura 7 muestra el modelo digital obtenido.

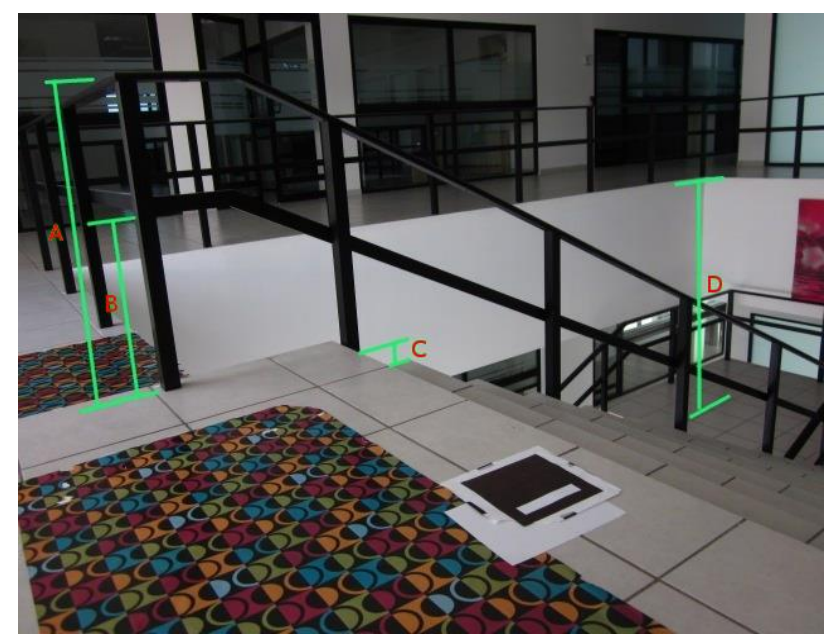

Figura 6 Escenario realista Fuente: Elaboración Propia

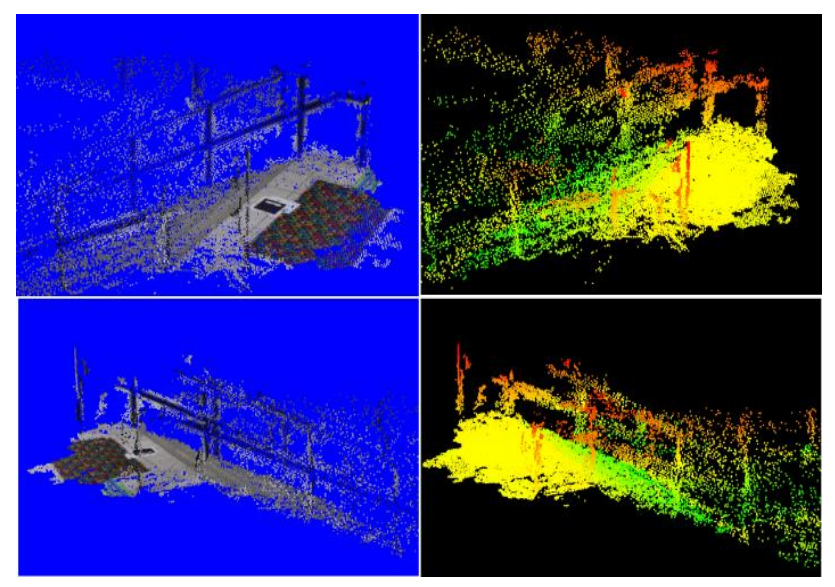

Figura 7 Modelo digital para el escenario realista Fuente: Elaboración Propia
Al igual que en el experimento de laboratorio, se evaluó estadísticamente la precisión considerando cuatro elementos de referencia. Los resultados obtenidos se resumen en la Tabla 2, con un error absoluto inferior a 9 $\mathrm{cm}$ para una altura real de $2.3 \mathrm{~m}$ (un error relativo inferior a $4 \%$ ).

\begin{tabular}{|c|c|c|c|c|}
\hline $\begin{array}{l}\text { Altura } \\
\text { referencia }\end{array}$ & $\begin{array}{c}\text { Altura } \\
\text { real } \\
{[\mathrm{m}]}\end{array}$ & $\begin{array}{c}\text { Altura } \\
\text { estimada } \\
{[\mathrm{m}]}\end{array}$ & $\begin{array}{c}\text { Error } \\
\text { absoluto } \\
{[\mathrm{m}]}\end{array}$ & $\begin{array}{c}\text { Error } \\
\text { relativo } \\
{[\%]}\end{array}$ \\
\hline $\mathbf{A}$ & 0.920 & 0.8945 & 0.0255 & 2.77 \\
\hline B & 0.530 & 0.5344 & 0.0044 & 0.83 \\
\hline C & -0.170 & -0.1659 & 0.0041 & 2.41 \\
\hline D & -2.300 & -2.3823 & 0.0823 & 3.58 \\
\hline
\end{tabular}

Tabla 2 Resultados de estimación del modelo digital obtenido para el escenario realista

Fuente: Elaboración Propia

\section{Reconstrucción digital del terreno a posteriori}

Como se explicó anteriormente, el esquema propuesto actualiza de manera incremental el modelo digital del terreno, a medida que llegan bloques de 3 imágenes consecutivas. Este modelo incremental tiene la precisión suficiente para permitir su utilización para la toma de decisiones en línea, es decir, al mismo tiempo que se sobrevuela el terreno.

Sin embargo, con este esquema también es posible hacer una reconstrucción a posteriori, es decir, cuando se ha completado el sobrevuelo del terreno y se cuenta con el total del conjunto de imágenes. De esta manera, se obtiene un modelo digital muy preciso, más denso y sin puntos espurios, pero que requeire un tiempo mucho más importante para su construcción (algunos minutos).

La Figura 8 muestra el modelo digital obtenido al procesar, a posteriori, el conjunto completo de 72 imágenes obtenidas durante uno de los sobrevuelos. Se puede observar la mayor densidad y precisión del modelo, con errores relativos inferiores a $1.5 \%$ en la altura máxima de la maqueta. 

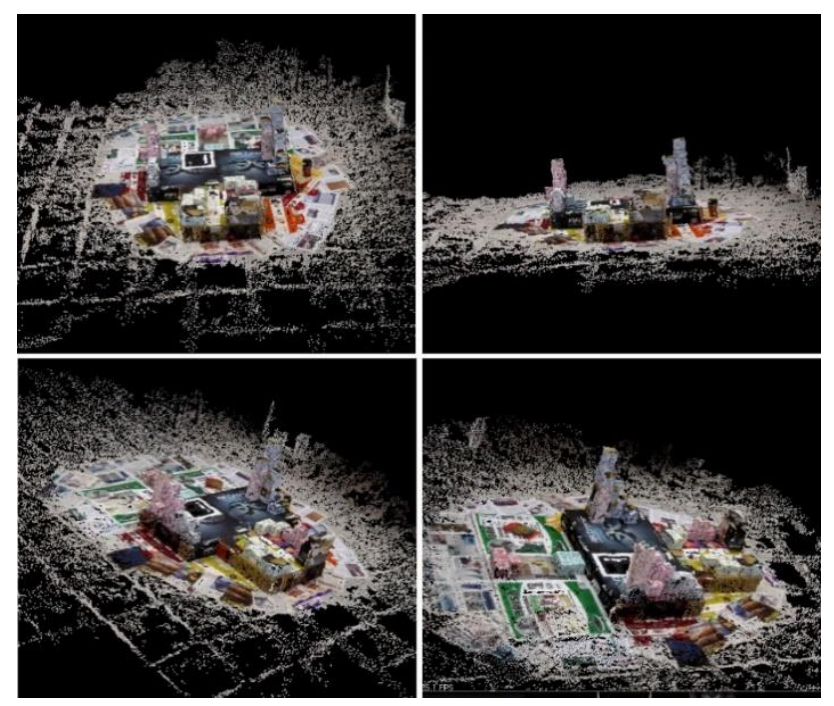

Figura 8 Modelo digital obtenido a posteriori

Fuente: Elaboración Propia

\section{Conclusión}

Una primera conclusión a la que llegamos, durante la experimentación realizada, es que la calidad de las imágenes tiene un impacto directo en la calidad del modelo digital obtenido. Es importante considerar esta situación al elegir la cámara a utilizar.

Pero más allá de esta consideración, este trabajo muestra que es posible obtener un modelo digital del terreno, de manera incremental (al mismo tiempo que se sobrevuela el medio ambiente), lo suficientemente preciso que pueda utilizarse para la toma de decisiones (navegación, navegación autónoma, por mencionar algunos), empleando herramientas asequibles: una computadora de escritorio y un microdron comercial de bajo costo. Como no se utiliza información georreferenciada, es posible desarrollar aplicaciones en interiores y zonas donde la señal GPS no está disponible. De esta manera, el esquema propuesto puede ser una herramienta que permita, tanto a científicos como a profesionales, abordar problemas complejos que requieran el acceso a un modelo del terreno que se construye al mismo tiempo que se sobrevuela el medio ambiente.

\section{Agradecimiento}

Los autores desean expresar su agradecimiento al Conacyt y al Cinvestav Tamaulipas por las facilidades prestadas durante el desarrollo de este trabajo. Asimismo, desean expresar también su reconocimiento a los autores de las bibliotecas de software libre empleadas en el desarrollo de este trabajo: OpenMVG, OpenCV, PMVS2 y Point Cloud Library.

\section{Referencias}

Barazzetti, L.; Scaioni, M. Orientation And 3D Modelling From Markerless Terrestrial Images: Combining Accuracy With Automation. The Photogrammetric Record 2013, 25, 2010.

Besnerais, G.; Sanfourche, M.; Champagnat, F. Dense height map estimation from oblique aerial image sequences. Computer Vision and Image Understanding 2008, 109, 204-225.

Bills, C.; Chen, J.; Saxena, A. Autonomous MAV flight in indoor environments using single image perspective cues. International Conference on Robotics and Automation (ICRA), 2011.

Bybee TC, Budge SE. Textured digital elevation model formation from low-cost UAV LADAR/digital image data. Proceedings of SPIE - the International Society for Optical Engineering, 2015, paper 131.

Call, B. Obstacle Avoidance For Unmanned Air Vehicles. Master's thesis, Brigham Young University, 2006.

Chen, H.; Bhanu, B. 3D free-form object recognition in range images using local surface patches. Pattern Recognition Letters 2007, 28, 1252-1262.

Davison, A. Real-Time Simultaneous Localization and Mapping with a Single Camera. 2003. Proceedings. Ninth IEEE International Conference on Computer Vision, 2003, Vol. 2, pp. 1403 - 141.

Engel, J.; Sturmand, J.; Cremers, D. CameraBased Navigation of a Low-Cost Quadrocopter. Intelligent Robots and Systems (IROS), 2012 IEEE/RSJ International, 2012, Vol. 320.

Furukawa, Y.; Ponce, J. Accurate, dense, and robust multi-view stereopsis. Pattern Analysis and Machine Intelligence 2010, 32, 1362-1376.

Garrido-Jurado, S.; Muñoz-Salinas, R.; MadridCuevas, F.; Marín-Jiménez, M. Automatic generation and detection of highly reliable fiducial markers under occlusion. Pattern Recognition 2014, 47, 2280 - 2292.

Gertler J. US Unmanned Aerial Systems. Library of Congress Washington DC Congressional Research Service. 2012.

RAMÍREZ-TORRES, José Gabriel \& LARRAÑAGA-CEPEDA, Ander. Empleo de microdrones para la reconstrucción tridimensional del terreno. Revista de Ingeniería Civil. 2019 
Harris, C.; Stephens, M. A combined corner and edge detector. Proceedings of the 4th Alvey Vision Conference, 1988, pp. 147-151.

Haubeck K, Prinz T. A UAV-based low-cost stereo camera system for archaeological surveys - experiences from Doliche (Turkey). International Archives of the Photogrammetry, Remote Sensing and Spatial Information Sciences. 2013. 40(1W2) 195-200.

Hough, P. Method and means for recognizing complex patterns. US 3069654, 1962.

Jimenez-Lugo, J.; Zell, A. Framework for Autonomous Onboard Navigation with the AR.Drone. International Conference on Unmanned Aircraft Systems 2013, pp. 575-583.

Khoshelham, K. Extending generalized Hough transform to detect $3 \mathrm{~d}$ objects in laser range data. Proc. ISPRS Workshop on Laser Scanning, 2007, pp. 206-210.

Kršák B, Blišt’an $\mathrm{P}$, Pauliková $\mathrm{A}$, Puškárová $\mathrm{P}$, Kovanič L, Palková J, Zelizňaková V. Use of low-cost UAV photogrammetry to analyze the accuracy of a digital elevation model in a case study. Measurement: Journal of the International Measurement Confederation, 2016, 91, 276-287.

Lamdan, Y.; Wolfson, H. On the error analysis of geometric hashing. Proc. IEEE Conf. on Computer Vision, 1991, pp. 22-27.

Lowe, D. Distinctive image features from scaleinvariant key points. International Journal of Computer Vision 2004, 60, 91-110.

Marr, D.; Hildreth, E. Theory of Edge Detection. Proceedings of the Royal Society of London, 1980, pp. 215-217.

Mian, A.; Bennamoun, M.; Owens, R. On the Repeatability and Quality of Keypoints for Local Feature-based 3D Object Retrieval from Cluttered Scenes. International Journal of Computer Vision 2010, 89, 348-361.

Moulon, P.; Monasse, P.; Marlet, R. Adaptive Structure from Motion with a Contrario Model Estimation. ACCV 2012, 2012, Vol. 7727, pp. 257-270.
Nex F, Remondino F. UAV for 3D mapping applications: A review. Applied Geomatics, 2014, 6(1), 1-15.

Petrelli, A.; Stefano, L.D. On the repeatability of the local reference frame for partial shape matching. 13th International Conference on Computer Vision (ICCV), 2011, pp. 2244 2251.

Pollefeys, M.; Nistér, D.; et al. Detailed RealTime Urban 3D Reconstruction from Video. International Journal of Computer Vision 2008, $78,143-167$.

Sanfourche M, et al. Perception for UAV: Vision-Based Navigation and Environment Modeling. Journal Aerospace Lab 2012, 4, 119.

Scaramuzza, D.; Fraundorfer, F.; Pollefeys, M.; Siegwart, R. Absolute scale in structure from motion from a single vehicle mounted camera by exploiting non holonomic constraints. 2009 IEEE 12th International Conference on Computer Vision, 2009, pp. 1413 - 1419.

Sirmacek B, d'Angelo P, Krauss T, Reinartz P. Enhancing Urban Digital Elevation Models Using Automated Computer Vision Techniques. International Conference on Pattern Recognition, 2010.

Soto-Guerrero D, Ramírez-Torres JG. A Human-Machine Interface with Unmanned Aerial Vehicles. Proceedings of the 10th International Conference on Electrical Engineering, Computing Science and Automatic Control (CCE '13), 2013, pp. 307-312.

Tombari, F.; S.Salti.; DiStefano, L. Unique signatures of histograms for local surface description. ECCV'10 Proceedings of the 11th European conference on computer vision conference on Computer vision, 2010, pp. 356369.

Weibel, R.; M.Heller. Digital Terrain Modelling. 1993.

Weiss, S.; Achtelik, M.; Kneip, L.; Scaramuzza, D.; Siegwart, R. Intuitive 3D Maps for MAV Terrain Exploration and Obstacle Avoidance. Journal of Intelligent and Robotics Systems 2011, 61, 473-493. 
Wen-Chung, C.; Shu-An, L. Real-time featurebased 3D map reconstruction for stereo visual guidance and control of mobile robots in indoor environments. 2004 IEEE International Conference on Systems, Man and Cybernetics, 2008, Vol. 6, pp. 5386 - 5391.

Zhong, Y. Intrinsic shape signatures: A shape descriptor for 3D object recognition. Proc. 3DRR Workshop (in conj. with ICCV), 2009, pp. $689-696$. 